\title{
A comprehensive analysis of $R H O A$ mutation positive and negative angioimmunoblastic T-cell lymphomas by targeted deep sequencing, expression profiling and single cell digital image analysis
}

\author{
ALEXANDRA BUTZMANN $^{1,2}$, KAUSHIK SRIDHAR $^{1,2}$, DIWASH JANGAM $^{1}$, JYOTI KUMAR $^{1}$, \\ MALAYA KUMAR SAHOO ${ }^{1}$, NAHID SHAHMARVAND ${ }^{1}$, ROGER WARNKE ${ }^{1}$, ELUMALAI RANGASAMY ${ }^{3}$, \\ BENJAMIN ALAN PINSKY ${ }^{1}$ and ROBERT SHIGEO OHGAMI ${ }^{1,2}$ \\ ${ }^{1}$ Department of Pathology, Stanford University, Stanford, CA 94305; ${ }^{2}$ Department of Pathology, \\ University of California, San Francisco, CA 94143; ${ }^{3}$ Agilent Technologies, Santa Clara, CA 95051, USA
}

Received October 1, 2019; Accepted April 22, 2020

DOI: $10.3892 / \mathrm{ijmm} .2020 .4686$

\begin{abstract}
Angioimmunoblastic T-cell lymphoma (AITL) is a uniquely aggressive mature T-cell neoplasm. In recent years, recurrent genetic mutations in ras homolog family member A (RHOA), tet methylcytosine dioxygenase 2 (TET2), DNA methyltransferase 3 alpha (DNMT3A) and isocitrate dehydrogenase $[\mathrm{NADP}(+)] 2(I D H 2)$ have been identified as associated with AITL. However, a deep molecular study assessing both DNA mutations and RNA expression profile combined with digital image analysis is lacking. The present study aimed to evaluate the significance of molecular and morphologic features by high resolution digital image analysis in several cases of AITL. To do so, a total of 18 separate tissues from 10 patients with AITL were collected and analyzed. The results identified recurrent mutations in RHOA, TET2, $D N M T 3 A$, and $I D H 2$, and demonstrated increased DNA mutations in coding, promoter and CCCTC binding factor (CTCF) binding sites in RHOA mutated AITLs vs. RHOA non-mutated cases, as well as increased overall survival in $R H O A$ mutated patients. In addition, single cell computational digital image analysis morphologically characterized $R H O A$ mutated AITL cells as distinct from cells from RHOA mutation negative patients. Computational analysis of single cell morphological parameters revealed that $R H O A$ mutated cells have decreased eccentricity (more circular) compared with $R H O A$ non-mutated
\end{abstract}

Correspondence to: Dr Alexandra Butzmann or Dr Robert Shigeo Ohgami, Department of Pathology, University of California, 513 Parnassus Avenue, Room HSW 450, San Francisco, CA 94143, USA

E-mail: alexandra.butzmann@ucsf.edu

E-mail: robert.ohgami@ucsf.edu

Key words: angioimmunoblastic T-cell lymphoma, peripheral T-cell lymphoma, ras homolog family member A
AITL cells. In conclusion, the results from the present study expand our understanding of AITL and demonstrate that there are specific cell biological and morphological manifestations of $R H O A$ mutations in cases of AITL.

\section{Introduction}

T-cell lymphomas (TCL) are rare hematolymphoid malignancies with poor overall survival. A major subtype of TCL, angioimmunoblastic T-cell lymphoma (AITL), has been the focal point of numerous studies. AITL is characterized by unique clinical, morphologic, immunophenotypic and molecular features. AITL possesses a characteristic background of increased vascularity along with morphologically atypical T-cells that present with a follicular helper T-cell immunophenotype (1-3).

In recent years, broad genetic profiling of AITL reported that it often harbors mutations in ras homolog family member A (RHOA; $G 17 \mathrm{~V})$, tet methylcytosine dioxygenase 2 (TET2), DNA methyltransferase 3 alpha $(D N M T 3 A)$ and isocitrate dehydrogenase [NADP(+)] 2 (IDH2) (4-9). For example, RHOA G17V mutations have been the focus of intense research. RHOA G17V mutations are believed to be highly associated with the most classic cases of AITL (10) and are found in $50-80 \%$ of AITL cases (7). In addition, RHOA mutated cases are believed to be characterized by increased microvascular density and to express a high number of follicular helper T-cell markers (10).

To better understand the pathophysiology of AITL, the present study performed targeted deep sequencing on 18 tissues samples from 10 patients with AITL, which were biopsied at the time of diagnosis. Both targeted DNA sequencing and RNA-sequencing, including single nucleotide polymorphisms (SNPs) and insertions/deletions (indel) analysis, translocation analysis, and gene expression as well as pathway analysis were performed on AITL cases. Computational image segmentation and analysis from haematoxylin and eosin (H\&E) stained sections were completed in order to quantify and differentiate 
morphological parameters between the RHOA mutated and $R H O A$ non-mutated cases. In addition, this analysis was coupled to the outcomes of patients to compare overall survival.

\section{Materials and methods}

Patient cohort. In the present study, 10 cases of AITL were selected from the archives (July 2004-October 2018) of the Department of Pathology, Stanford University Medical Center (Stanford, CA) where adequate tissues were available and diagnoses could be confirmed. Patient medical record charts, clinical and laboratory data, treatment data and slides [formalin fixed paraffin embedded (FFPE) lymph node tissues, clinically stained] were reviewed, and diagnoses were confirmed by RO, JK, AB and RW according to the 2016 World Health Organization (WHO) $(11,12)$ criteria. In total, 18 tissue samples were analyzed. H\&E stained slides were generated by staining FFPE tissues slices of $4-\mu \mathrm{m}$ thickness. Auto-staining was performed on a Leica Autostainer XL according to manufacturer's instructions (Leica Microsystems, Inc.). This study received ethical approval from Stanford University's Institutional Review Board (reference no. IRB-22359).

Targeted DNA deep sequencing. Targeted sequencing was performed as previously described (13). Briefly, DNA was extracted from FFPE lymph node tissues using the DNA Storm FFPE DNA Extraction kit (Cell Data Sciences). Quality and quantity of extracted nucleic acids was assessed by Qubit (Thermo Fisher Scientific, Inc.) and the 2100 Bioanalyzer (Agilent Technologies, Inc.). For targeted next-generation sequencing (NGS), our Heme Malignancy Evaluation and Infectious Disease panel (HeME-ID; Table SI) was used, which targets 354 genes mutated in hematolymphoid diseases and allows identification of point mutations, insertion/deletions (indels) and translocations, as well as 13 viruses and bacteria associated with hematolymphoid diseases. DNA (150 ng) was used to prepare the DNA library using the SureSelectXT HS enrichment kit (Agilent Technologies, Inc.). A 100 base-pair paired end high-throughput sequencing was performed on a HiSeq4000 platform (Illumina, Inc.) at an average depth of 1000 fold. For downstream processing of the output files, Genome Analysis Toolkit (GATK; https://gatk.broadinstitute. org/hc/en-us) best practices for alignment, single-nucleotide variant and structural variant analysis were followed (14), BWA-MEM algorithm for alignment was used and further analysis was performed using GATK (version 4.0; https://gatk. broadinstitute.org/hc/en-us), Varscan2 (version v2.3.8) (15), and SNNPET (Agilent Technologies, Inc.). For variant calling, SureCall (version 4.1; Agilent Technologies, Inc.) was used and mutations were analyzed with a variant allele frequency at $\geq 2 \%$ in single sample analysis mode, which was justified by a high read depth and the use of molecular barcodes in the Sureselect ${ }^{\mathrm{XT} \text { HS}}$ kit. Filters were applied following the mutation caller's recommendations. In order to call a mutation, a 200x read coverage per base, a minimum coverage in forward and reverse direction and a maximum allele frequency of $40 \%$ were required and a minimum Combined Annotation Dependent Depletion (CADD) score of 20. The same filters were applied for small indels analysis. For annotation, SureCall and Seattleseq
(University of Washington, Seattle; version 9.10) were used. For further curation of the variants, Exome Aggregation Consortium (16), Clinvar (17), 1000 genomes (18), Exome Variant Server (19) and Varsome (20) were used. For further downstream analysis, the MutationalPatterns (21) Package from $\mathrm{R}$ was used [mutational signatures analysis using COSMIC signatures, enrichment/depletion analysis of promoter regions, CTCF (CCCTC-binding factor) bindings sites and promoter flanking regions], and Pathway Analysis was done with EnrichR (22), Gene Set Enrichment Analysis $(23,24)$ and ConsensusPathDB-human (25). Evaluation of microorganisms was performed using the subtraction method as previously described for shotgun metagenomic sequencing $(26,27)$. For viruses, results were interpreted by percent coverage of the targeted regions and average depth. Samples were classified as follows: Negative, equivocal and positive. A positive result required all three targeted regions of a sample to have a minimum coverage of $75 \%$ and an average depth of at least 5 . An equivocal result indicated that all three targeted regions had a $10-75 \%$ coverage with a minimum average read depth of 1 . A negative result indicated all other scenarios.

RNA-sequencing and data analysis. RNA was extracted from FFPE lymph node tissues using the RNA Storm FFPE DNA Extraction kit (Cell Data Sciences). Quality and quantity of extracted nucleic acids was assessed by Qubit analysis and 2100 Bioanalyzer. RNA (200 ng) was used to prepare RNA libraries with the SureSelect ${ }^{\mathrm{XT}}$ RNA Direct kit (Agilent Technologies, Inc.; with Sureselect Exome V6 + UTR, capture library) for strand-specific sequencing libraries. A 150 base-pair paired end whole transcriptome sequencing was performed on a high-throughput sequencing HiSeq4000 platform with a mean coverage of 300 million reads. For downstream processing of our output files, Hisat2 (version 2.1.0) (28) was used for alignment and HT-Seq (version 0.11.1) (29) was used for generation of the count files. Gene expression analysis was performed using RStudio (version 3.5.3) (30). Exploratory analysis [Principal Component Analysis (PCA) and heatmap with hierarchical clustering by Euclidean distance] was performed using ClustVis (31). For differential gene expression, the DeSeq and EdgeR packages were used. The immune cell composition was analyzed using CIBERSORT (https://cibersort.stanford.edu/), which is a tool that uses the constellation of expressed genes, based on known signatures, in order to refer to the suspected immune cell composition within the analyzed sample (32).

Statistical analysis. Statistical analysis was performed using R (Cran; version 3.6.0; https://cran.r-project.org/) and RStudio (RStudio Inc., version 1.2.1335; https://rstudio.com/). Student's t-test and Mann-Whitney U test were performed to evaluate differences between the mutational burden of RHOA positive and negative cases, the average CADD score of RHOA positive and negative cases, the mutational burden of EBV positive and negative cases and the average CADD score of EBV positive and negative cases. $\chi^{2}$ test and Kaplan Meier Survival analysis were also used. Statistical significance was determined with the Log-rank. A two-sided binomial test was used to compare the two categories of RHOA positive and negative cases with regards to expected vs. observed mutational burdens in 
Table I. Clinicopathological characteristics of the 10 patients with angioimmunoblastic T-cell lymphoma.

\begin{tabular}{|c|c|c|c|c|c|c|c|}
\hline $\begin{array}{l}\text { Case } \\
\text { number }\end{array}$ & $\begin{array}{l}\text { Age, } \\
\text { years }\end{array}$ & Sex & Histology & $\begin{array}{l}\text { RHOA } \\
\text { status }\end{array}$ & Treatment & $\begin{array}{l}\text { Time to } \\
\text { relapse, days }\end{array}$ & $\begin{array}{l}\text { Survival, } \\
\text { days }\end{array}$ \\
\hline Case 1 & 73 & Female & AITL+BCP & RHOA+ & $\begin{array}{l}\text { Prednisone, Chlorambucil, } \\
\text { Hydroxyurea, Rituximab }\end{array}$ & 1,364 & 3,449 \\
\hline Case 2 & 70 & Male & $\mathrm{AITL}+\mathrm{BCP}$ & RHOA- & 6 cycles $\mathrm{R}-\mathrm{CHOP}+\mathrm{GCSF}$ & 310 & 1,177 \\
\hline Case 3 & 47 & Male & AITL+BCP & RHOA- & 1 cycle R-CHOP & 715 & 725 \\
\hline Case 4 & 49 & Male & AITL & RHOA- & NA & NA & NA \\
\hline Case 5 & 78 & Female & AITL & RHOA+ & Prednisone & 42 & 42 \\
\hline Case 6 & 59 & Male & AITL & RHOA- & 8 cycles $\mathrm{CHOP}$ & 181 & 181 \\
\hline Case 7 & 72 & Male & AITL & RHOA+ & Etanercept treatment & 1,005 & 1,005 \\
\hline Case 8 & 45 & Male & AITL & RHOA+ & $\begin{array}{l}6 \text { cycles } \mathrm{CHOP}+\text { Vincristine vs. } \\
\text { Brentuximab, auto-HCT }\end{array}$ & 1,733 & 1,733 \\
\hline Case 9 & 68 & Female & AITL & RHOA+ & $\begin{array}{l}\text { Steroids, } 1 \text { dose Cytoxan, } \\
\text { Romiplostim }+2 \text { doses IVIG, } \\
\text { cyclosporine treatment, } \\
\text { prednisone + IVIG continuously }\end{array}$ & No relapse & 1,839 \\
\hline Case 10 & 39 & Female & AITL & RHOA- & $\begin{array}{l}\text { SGN-35-014: } 4 \text { cycles CHOP + } \\
\text { Vincristine vs. Brentuximab, } \\
3 \text { cycles ICE+Brentuximab, } \\
\text { auto-HCT }\end{array}$ & 127 & 264 \\
\hline
\end{tabular}

AITL, angioimmunoblastic T-cell lymphoma; BCP, B-cell proliferation; LN, lymph node; BM, bone marrow; EBV, Epstein Barr Virus; F, female; M, male; N/A, not available; NED, no evidence of disease; R-CHOP, cyclophosphamide, doxorubicin, vincristine, and prednisone; HCT, hematopoietic cell transplant; IVIG, intravenous immunoglobulin; RHOA, ras homolog family member A.

defined genomic locations. $\mathrm{P}<0.05$ was considered to indicate a statistically significant difference.

Computational digital image analysis. H\&E images of lymph node sections were obtained using a conventional slide scanner (Leica Aperio AT2 slide scanner; Leica Microsystems, Inc.). The H\&E images were patched at an optical zoom of $x 40$, uniform width of 68 pixels and height of 57 pixels. MATLAB program (version 2019b; https://www.mathworks.com/) was used for image analysis wherein the cells of interest were segmented based on their pixel intensities (33). The unsharp filter in the Image Processing Toolbox was used to improve the edge contours and contrast among the different cells of interest. Based on the input pixels, cells were clustered using $k$-means clustering $(34,35)$. For an input image $\mathrm{I}(\mathrm{x}, \mathrm{y})$, the distance between each cluster center, $\mathrm{c}_{I(I=1: \mathrm{n})}$, and the corresponding data points is denoted by the equation 1 :

$$
d=\left|I_{(x, y)}-c_{I}\right|
$$

This iterative algorithm seeks to minimize the sum of all distances (d) between all the data points in a particular cluster to its cluster centroid and is calculated using the equation 2 :

$$
c_{I}=\frac{1}{k} \sum_{y \in c_{l}} \sum_{x \in c_{I}} I(x, y)
$$

Once the necessary cluster that best matched the target cell body was identified, the respective image was transformed into its corresponding grayscale format. Finally, the regionprops function from MATLAB was used to extract eight parameters, including circularity, area (in pixel $^{2}$ ), major axis, minor axis, eccentricity, equivalent diameter $\left[\sqrt{ }\left(4 * \frac{\text { area }}{\pi}\right)\right]$, solidity (measure of the indentations on a cell surface) and perimeter (in pixels). Since it has been reported that cancer cells exhibit an increased variability in roundness, elongation and differences in nuclear shape $(36,37)$, cells were classified based on these parameters. The resulting datasets of $R H O A$ mutation positive and $R H O A$ mutation negative samples were compared using Principal Component Analysis (PCA) in R studio [R version 3.5.1 (2018-07-02); https://rstudio.com/] to quantify the deviation between them and identify the most variable parameters between the samples.

\section{Results}

Patient cohort. Tissue biopsies from 10 patients with AITL were selected in the present study. All cases were reviewed by RO, JK, AB and RW and classified according to the 2016 revised WHO classification. The 10 patients consisted of four women and six men (average age, 60 years; age range, 39-78 years). Five patients were positive for Epstein-Barr virus $(E B V)$ infected cells following analysis by in situ hybridization (ISH). Five patients were RHOA mutation positive and five patients were $R H O A$ mutation negative. Treatment regimens are presented in Table I. Among the 10 patients, only one (case 9) did not relapse following initial therapy.

DNA mutational analysis demonstrates differential mutational profiles in RHOA positive cases vs. RHOA negative 


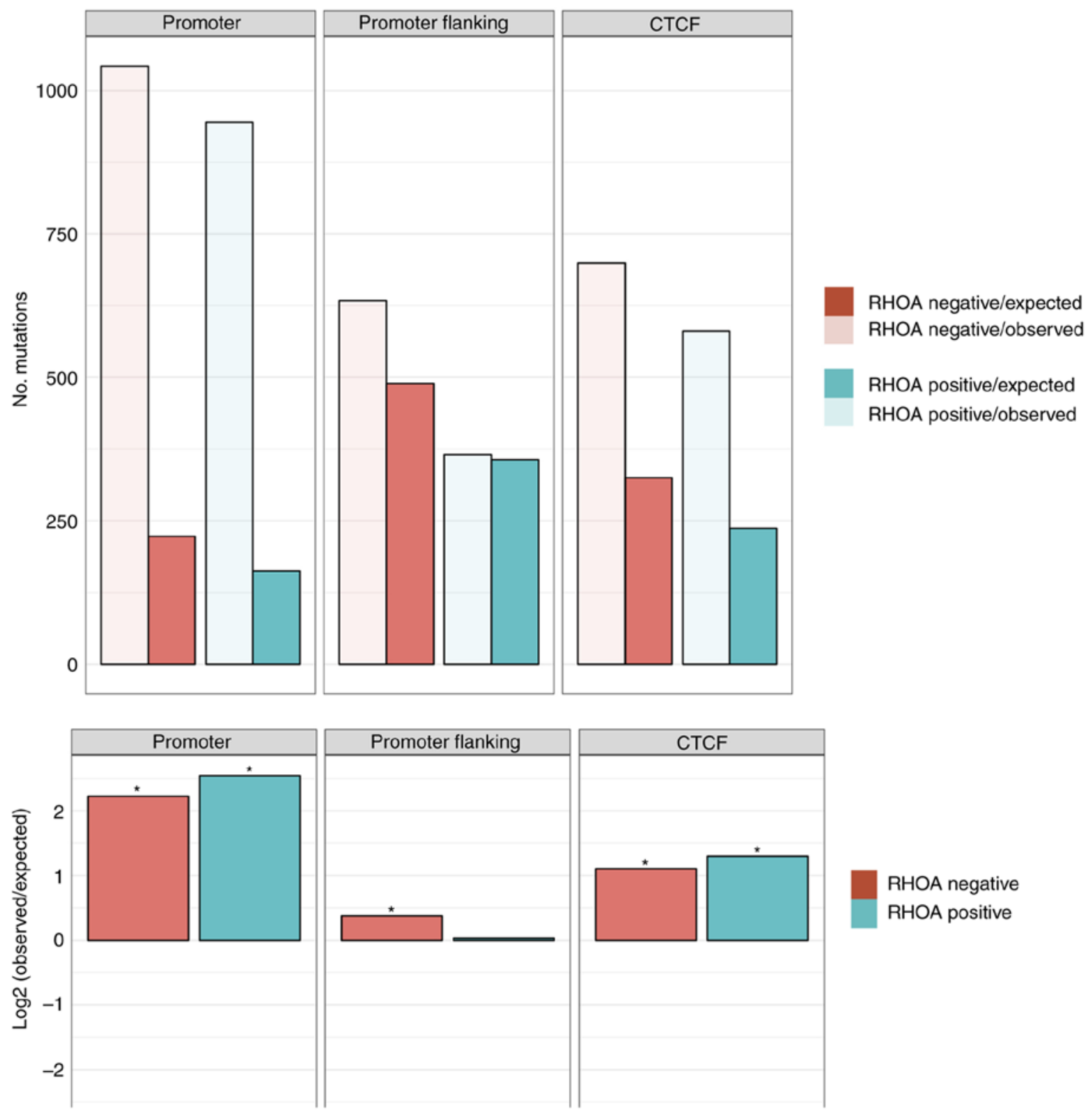

Figure 1. Enrichment/depletion analysis of the mutational burden in defined genomic regions for the RHOA positive (green) cases vs. $R H O A$ negative (red) cases with the number of observed mutations (transparent) vs. the number of expected mutations (solid) in the upper panel. The ratio of observed/expected on a $\log 2$ rank is depicted in the lower panels of the figure and an asterisk indicates significance $(\mathrm{P}<0.05$; two-sided binomial test). AITL, angioimmunoblastic T-cell lymphoma; RHOA, ras homolog family member A.

cases. Targeted DNA deep sequencing was performed on all cases to understand the pathophysiology of AITL. The overall mutational burden ranged from 0-14. RHOA mutation positive cases showed a higher mutational burden (5.2 mutations/case) vs. $R H O A$ mutation negative cases (1.8 mutations/case) with mutations generally showing more deleterious changes in RHOA mutation positive cases (average CADD score of 24.9 vs. 21.8). The mutational profile in promoter regions and CTCF binding sites were also investigated and the results demonstrated that the mutational burden was higher than expected in these regions, especially for $R H O A$ mutation positive cases vs. the RHOA mutation negative cases (Fig. 1). Recurrent $I D H 2$, TET2 and DNMT3A mutations have also been reported in AITL cases. We identified IDH2 R172 mutations in three of our cases (cases 5, 8 and 9). Two of these cases also showed the recurrent TET2 G422Efs*5 mutation (Fig. 2). The results demonstrated that TET2 was mutated in four additional samples. In addition, other genes known to be mutated in TCLs were mutated in our cases, and $C D 28$ was mutated in one case (case 9) and DNMT3A was mutated in another case (case 5). Furthermore, ETV6, EP300, STAT3, JAK2, FYN and PLCG1 genes were mutated in the samples analyzed in the present study. The HeME-ID Panel can evaluate common breakpoints and translocations, including for vav guanine nucleotide exchange factor 1 (VAVI; Table SI). No translocations were identified in the samples (data not shown). Finally, the absolute contribution of the COSMIC signatures (version 2), which are cancer-type specific signatures (liver, lung, stomach, B-cell lymphomas), was also investigated in the AITL samples with the Mutational Patterns package in R. However, no major contribution of a mutational signature in the AITL cases was identified. The mutational signature amongst all cases was the 
Table II. Overview of EBV in AITL cases.

\begin{tabular}{|c|c|c|c|}
\hline Case & Tissue & $\begin{array}{l}\text { EBV infected cells } \\
\text { (by EBER ISH) }\end{array}$ & EBV NGS \\
\hline Case 1 & LN & Negative & Negative \\
\hline Case 2 & LN & Positive & Equivocal \\
\hline \multirow[t]{2}{*}{ Case 3} & LN & Negative & Negative \\
\hline & BM & & Negative \\
\hline \multirow[t]{2}{*}{ Case 4} & LN & Positive & Positive \\
\hline & BM & Negative & Negative \\
\hline Case 5 & LN & Negative & Negative \\
\hline \multirow[t]{2}{*}{ Case 6} & $\mathrm{LN}$ & $\mathrm{N} / \mathrm{A}$ & Negative \\
\hline & BM & & Negative \\
\hline \multirow[t]{2}{*}{ Case 7} & $\mathrm{LN}$ & Positive & Positive \\
\hline & $\mathrm{BM}$ & & Negative \\
\hline \multirow[t]{2}{*}{ Case 8} & $\mathrm{LN}$ & Positive & Positive \\
\hline & BM & & Negative \\
\hline Case 9 & LN & Negative & Equivocal \\
\hline \multirow[t]{4}{*}{ Case 10} & $\mathrm{LN}$ & Positive & Positive \\
\hline & BM & N/A & Negative \\
\hline & BM & Positive & Equivocal \\
\hline & BM & Negative & Negative \\
\hline
\end{tabular}

EBV, Epstein Barr Virus; N/A, not available; NGS, next generation sequencing. $\mathrm{LN}$, lymph node. BM, bone marrow.

same with a majority of $\mathrm{C}>\mathrm{T}$ and $\mathrm{T}>\mathrm{C}$ base exchanges independent from RHOA mutation status or tissue type (Fig. S1).

EBV infection in AITL tissues. Five out of 10 patients had EBV infected cells by Epstein-Barr encoding region ISH (Table II). EBV and 13 other microorganisms as well as 354 human genetic mutations were targeted by using our targeted panel and sequencing approach. We performed targeted deep sequencing with our HeME-ID panel on our tissue samples directly and detected EBV in 4 cases as positive and 2 cases as equivocal (Table SII). All matched bone marrow samples were negative for EBV infection based on our criteria, apart from case 10 which had equivocal EBV infection of the bone marrow. Table II represents the viral findings from immunohistochemistry compared with results from NGS. No difference in the gene expression pattern was observed between EBV positive and EBV negative cases. The genomic analysis showed a higher mutational burden in EBV negative cases (average 8.25 vs. average 4 in EBV positive); however, a higher CADD score in mutated genes in EBV positive cases was observed (average CADD score 27.11 vs. average CADD score 17.7 in EBV negative). Survival analysis showed no difference between EBV infected and non-infected cases (Fig. S2).

RNA expression profiling identifies an altered immunologic environment in all AITL cases. Investigation of the associated immune system based on the gene expression profile of the AITL cases demonstrated that the majority of infiltrating immune cells consisted of naïve B-cells (yellow, Fig. 3) and resting memory CD4 T-cells (beige, Fig. 3). The third largest component in our AITL cases were the T-follicular helper cells (green, Fig. 3). Fig. 3 is a representation of the contribution of the immune cell expression patterns analyzed by the CIBERSORT pipeline. RHOA positive cases are circled in red. There is no difference in the immunologic background of RHOA mutation positive compared with $R H O A$ mutation negative cases.

AITL cases form two groups independent from RHOA mutational status or EBV infection status based on gene expression analysis. Following gene expression analysis, AITL cases were separated into two clusters. Fig. 4 represents a heatmap of the gene expression in our AITL samples. Hierarchical clustering was performed based on Euclidean distance. The results demonstrated that AITL samples were separated into two groups: Cases 3, 4, 5 and 6 in one group, and cases 1, 2, 7, 8 and 9 are in another group. Case 10 seemed to be an outlier with different expression patterns compared with the other samples. PCA was also performed on RHOA mutational status (Fig. S3) and the results confirmed similar sample grouping. However, no association to any of our tested conditions, such as RHOA status, EBV infection status or gender and age, was observed.

RHOA G17V mutated cases have better overall survival than RHOA mutation negative cases. Although treatments were different in our study group (Table I), the results from overall survival and relapse-free survival demonstrated that the RHOA mutation negative cases had a shorter relapse-free survival and showed a trend towards shorter overall survival (Fig. 5). Furthermore, we also performed Kaplan Meier analysis for EBV infection status of all cases, but no significant association between outcomes and EBV infection was identified (data not shown).

Computational single-cell image analysis separates neoplastic T-cells in RHOA mutated cases from neoplastic T-cells in RHOA wild type cases. It has been reported that RHOA mutations can affect tumorigenesis, in particular cell motility due to cellular processes associated with the formation of actin fibers and myosin activation (38). Subsequently, cell morphology of $R H O A$ mutation positive samples and RHOA mutation negative samples was evaluated.

The results from PCA of samples revealed that although the two subtypes, RHOA mutation positive and RHOA mutation negative, showed a maximum variance of $~ 53 \%$ along the first principal component (Figs. 6 and 7), there was an increased variation in the eccentricity of samples with $R H O A$ mutation positive cells (0.73) and RHOA mutation negative cells (0.74). This indicated that $R H O A$ mutation positive cells may be more circular compared with $R H O A$ mutation negative cells, which is explained by the reduced actin formation. Increased variation between these subtypes may be observed by increasing the total sample size and we hypothesize that greater variance may also be observed with respect to circularity $(36,37)$.

\section{Discussion}

In the present study, 10 cases of AITL were reported and compared for RHOA mutational status. Genomic analysis 


\begin{tabular}{|c|c|c|c|c|c|c|c|c|c|c|}
\hline & 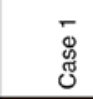 & $\begin{array}{l}N \\
刃 \\
\Phi \\
心 \\
心\end{array}$ & 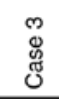 & 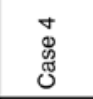 & $\begin{array}{l}0 \\
\Phi \\
\Phi \\
\widetilde{J} \\
0\end{array}$ & $\begin{array}{l}0 \\
\searrow \\
\mathbb{D} \\
心 \\
心\end{array}$ & $\begin{array}{l}\hat{\Phi} \\
\mathbb{8} \\
\mathbb{8}\end{array}$ & $\begin{array}{l}\infty \\
刃 \\
\Phi \\
心 \\
心\end{array}$ & $\begin{array}{l}\text { o) } \\
\Phi \\
\mathbb{J} \\
心\end{array}$ & $\begin{array}{l}\text { 으 } \\
\Phi \\
. \\
0 \\
0\end{array}$ \\
\hline RHOA & G17V & & & & G17V & & G17V & G17V & G17V & \\
\hline IDH2 & & & & & $\begin{array}{l}\text { R172 } \\
\end{array}$ & & & R172 & $\begin{array}{ll}\text { R172 } \\
\end{array}$ & \\
\hline TET2 & & R550* & & Q1274* & G422Efs"5 & & L34F & Q909* & G422Efs*5 & \\
\hline DNMT3A & & & & & D884N & & & & & \\
\hline CD28 & & & & & & & & & T195P & \\
\hline \multicolumn{11}{|l|}{ TP53 } \\
\hline ETV6 & & & & & R430* & & & & & \\
\hline EP300 & & & & C1745* & G1059E & R504W & & & & \\
\hline JAK2 & & & & & R225K & & & & & \\
\hline STAT3 & & & & & $\mathrm{R} 688 \mathrm{Q}$ & & & & & \\
\hline FYN & & & & & \begin{tabular}{|l|l|} 
L86I \\
\end{tabular} & & & & & \\
\hline PLCG1 & & & & & R778C & & & & & \\
\hline \multicolumn{11}{|l|}{ VAV1 } \\
\hline \multirow{2}{*}{\multicolumn{11}{|c|}{$\begin{array}{l}\text { CARD11 } \\
\text { PDGFRB }\end{array}$}} \\
\hline \multirow{2}{*}{\multicolumn{11}{|c|}{$\begin{array}{l}\text { PDGFRB } \\
\text { YTHDF2 }\end{array}$}} \\
\hline & & & & Q142* & & & & & & \\
\hline RAD21 & & & & & & & & & & \\
\hline \multicolumn{11}{|l|}{ MYC } \\
\hline \begin{tabular}{|l|} 
CNOT1 \\
\end{tabular} & R274C & & & & & & & & & \\
\hline \multicolumn{11}{|l|}{ CNOT3 } \\
\hline KCNH8 & & $\mathrm{D} 668 \mathrm{H}$ & & & $\overline{\mathrm{D} 47 \mathrm{~N}}$ & & & & & \\
\hline KIT & & & & & R942* & & & & & \\
\hline \multirow{2}{*}{\multicolumn{11}{|c|}{$\begin{array}{l}\text { RHOB } \\
\text { NUP214 }\end{array}$}} \\
\hline & & & & & & & & & & \\
\hline CD79A & & & & & G27S & & & & & \\
\hline EZH2 & & & & & Q102* & & & & & \\
\hline MAP2K1 & & & & & & C121S & & & & \\
\hline \multicolumn{11}{|l|}{ TET1 } \\
\hline TOP3A & & & & & & & & & & G721D \\
\hline \multicolumn{11}{|l|}{ GATA1 } \\
\hline STAT1 & & & & & & & & & E563K & \\
\hline CD58 & & & & & & & & & & $5158^{*}$ \\
\hline \multirow{2}{*}{\multicolumn{11}{|c|}{$\begin{array}{l}\text { JAK1 } \\
\text { PTPN1 }\end{array}$}} \\
\hline & & & & & & & & & & \\
\hline \multicolumn{11}{|l|}{\begin{tabular}{|l|} 
PTPRT \\
\end{tabular}} \\
\hline \multicolumn{11}{|l|}{ ZEB1 } \\
\hline B2M & & & & & & & & & & \\
\hline
\end{tabular}

Figure 2. Summary of the mutations found in AITL cases. Variants marked in red are pathogenic with a Combined Annotation Dependent Depletion (CADD) score $>30$. In green are the variants of unknown significance (VUS). Blue variants are deletions. AITL, angioimmunoblastic T-cell lymphoma.
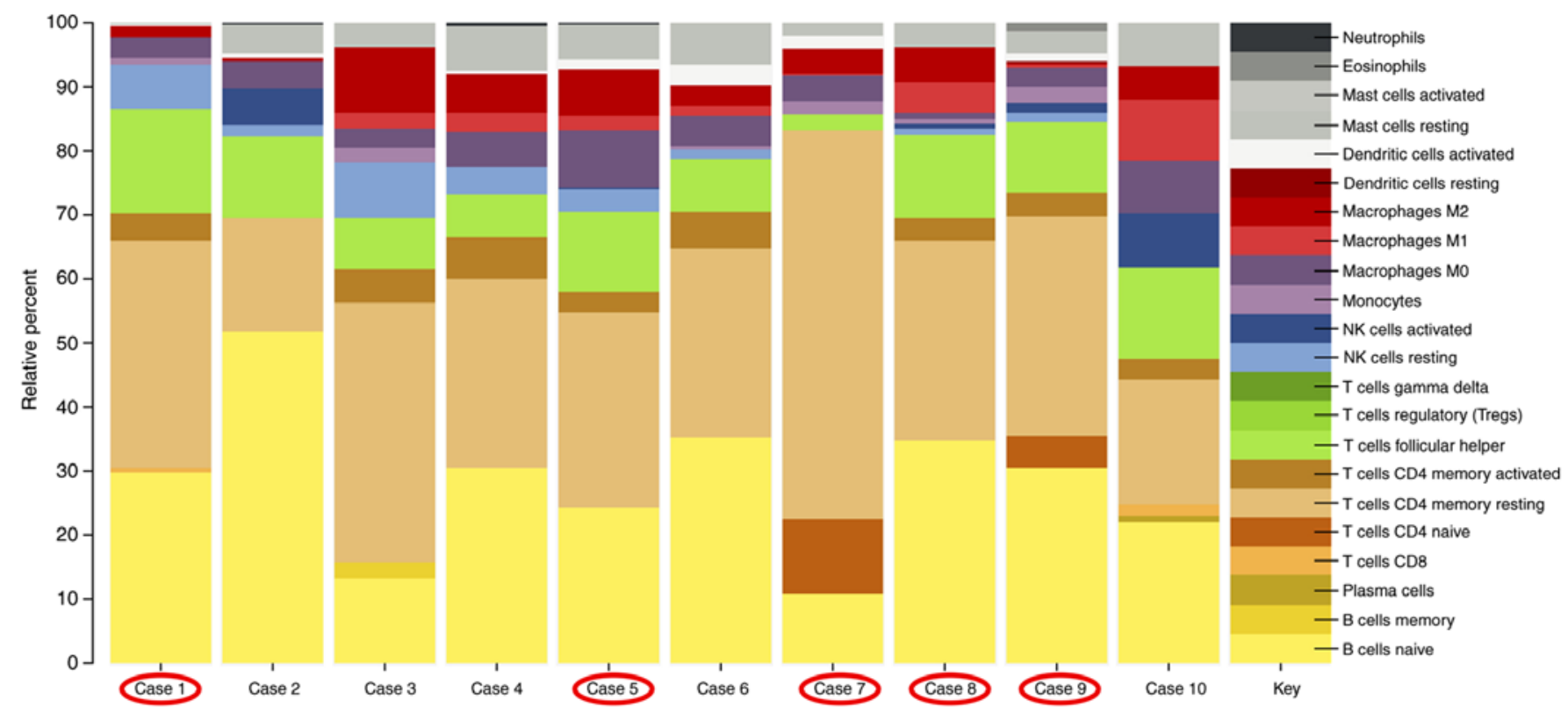

Figure 3. Immunologic cell subset pattern in RHOA mutated (circled in red) and RHOA non-mutated AITL cases. AITL, angioimmunoblastic T-cell lymphoma; RHOA, ras homolog family member A.

of the bone marrow samples for six of the cases was also performed. Although the present study was limited in size, only a few studies have compared RHOA mutation positive cases side-by-side with $R H O A$ negative cases $(10,39,40)$. The present study provided a broader genomic analysis coupled with computational digital image analysis. 


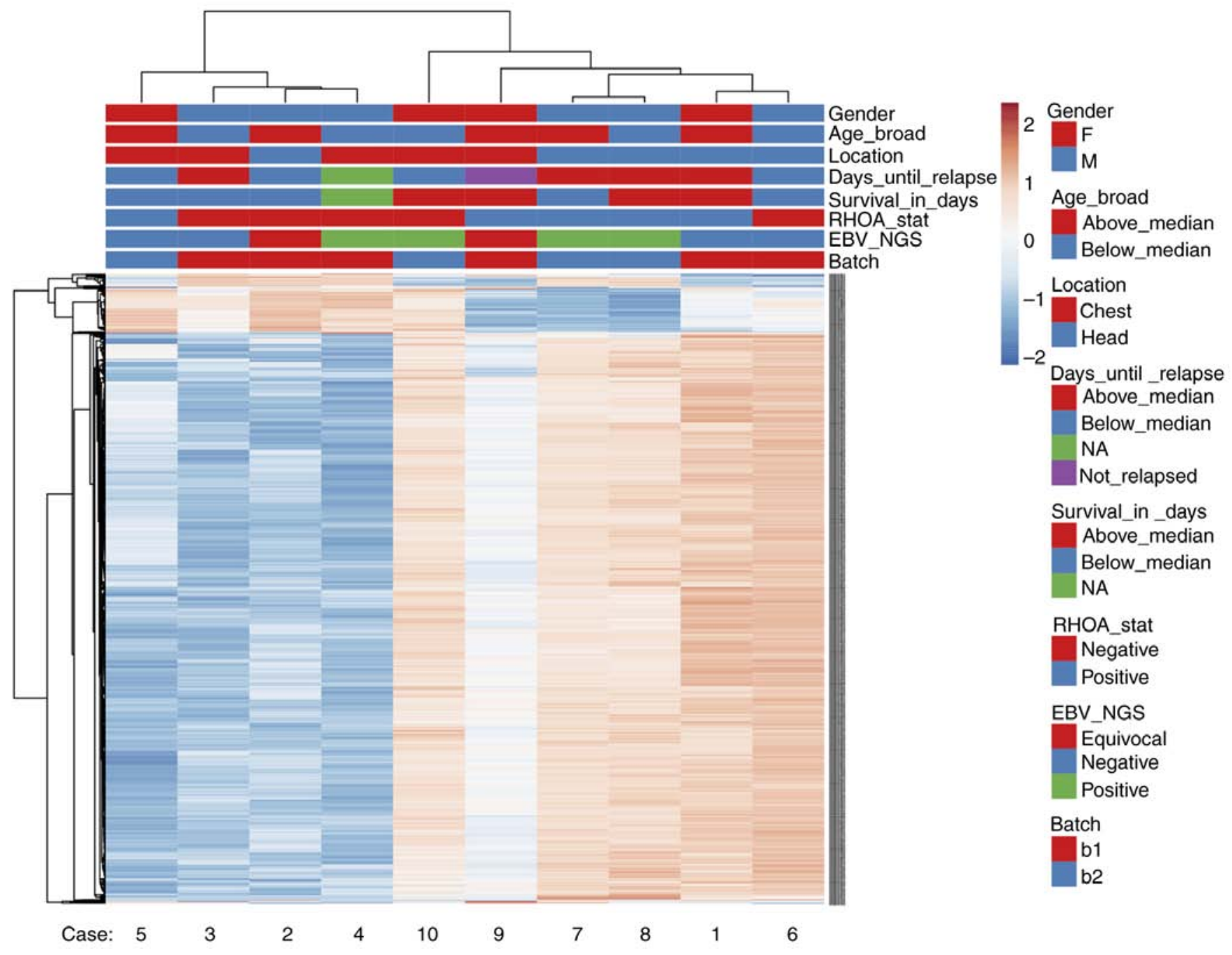

Figure 4. Heatmap with hierarchical clustering by Euclidean distance of the gene expression in all AITL cases. AITL, angioimmunoblastic T-cell lymphoma.
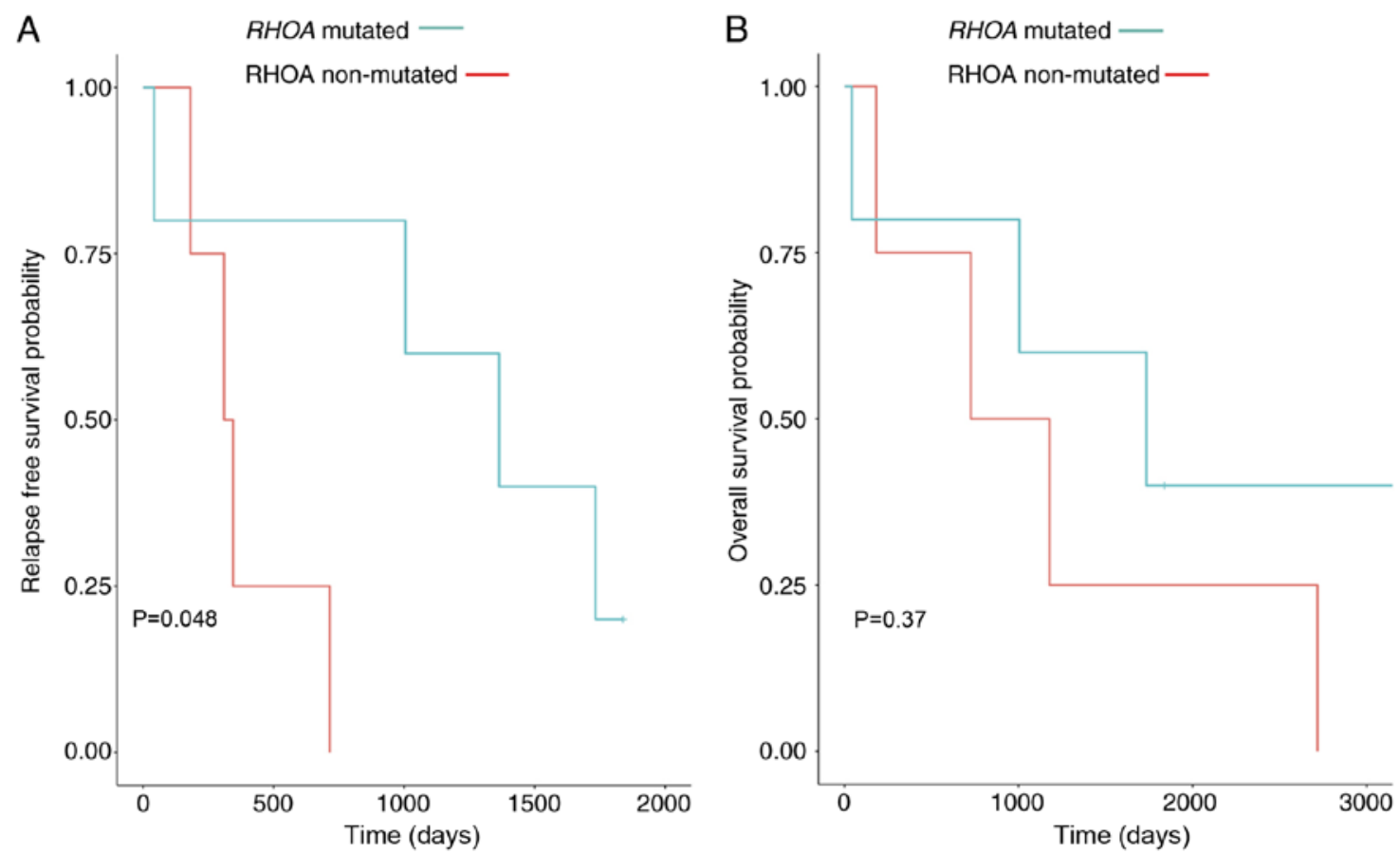

Figure 5. Kaplan-Meier survival analysis. (A) Relapse-free survival analysis. (B) Overall survival analysis. Red line is the RHOA mutation negative cases and the blue line represents the RHOA mutation positive cases. RHOA, ras homolog family member A. 


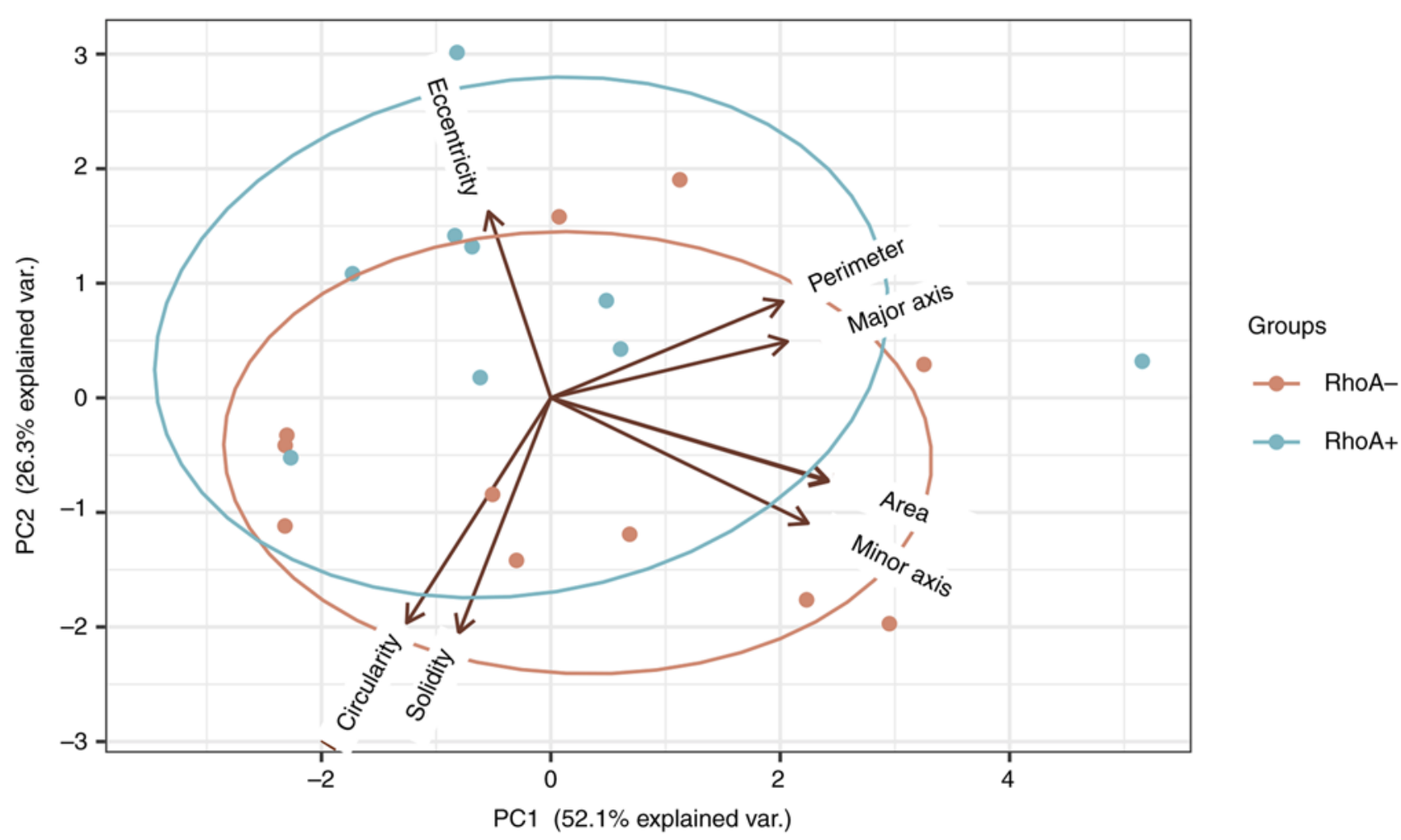

Figure 6. Principal Component Analysis of RHOA mutated and RHOA non-mutated AITL cells. Greatest variation was observed along the first Principal Component with reduced eccentricity in RHOA mutated AITL cases (0.73). AITL, angioimmunoblastic T-cell lymphoma; RHOA, ras homolog family member A.

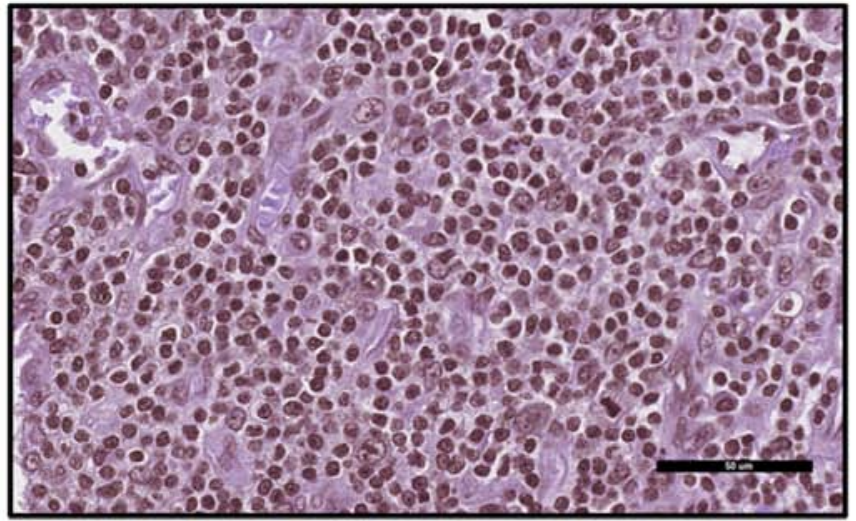

RhoA+

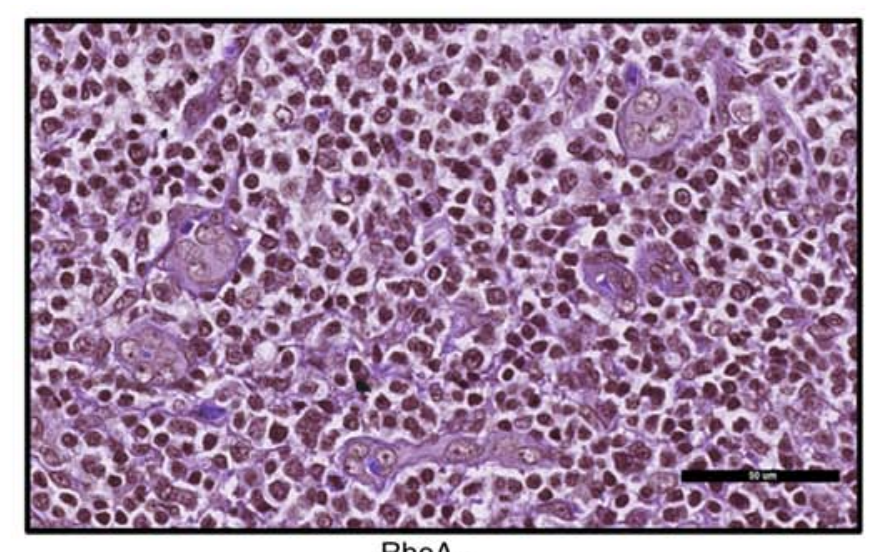

RhoA-

Figure 7. Haematoxylin and eosin images of RHOA+ and RHOA- samples (magnification, x40) converted to grayscale and clustered to identify morphological differences.

Recurrent RHOA G17V mutations have been previously identified in $>50 \%$ of AITL cases, and mutations in epigenetic regulators, including $I D H 2, T E T 2$ and DNMT3A, have also been described $(41,42)$. The present study also identified the frequently found mutations in AITL. The results also identified $I D H 2$ and TET2 mutations in association with the RHOA $G 17 \mathrm{~V}$ mutation. The role of $R H O A$ G17V mutations in combination with $I D H 2$ and TET2 within the pathogenesis of AITL has been previously studied as promoting T-follicular helper cell differentiation and as an important genetic hit for T-cell lymphomagenesis $(8,9,38,43)$. Other mutations that frequently occur in AITL, such as DNMT3A, VAVI, PLCG1, STAT3, $J A K 2$ and $F Y N$, are typically observed independently from
RHOA mutations (40). A previous study by Abate et al (44) also identified recurrent $V A V I$ translocations in peripheral TCL (PTCL). Our HeME-ID Panel also covered the breakpoint region of VAVI translocations; however, the present study did not identify any VAVI translocation in our cases. These differences could be due to the small sample size of AITL cases compared with the 152 PTCL samples (of which 60 were AITL samples) evaluated in the study by Abate et al.

Overall, RHOA mutation positive cases showed a more damaging mutational burden with an average CADD score of 24.9 compared with 21.8 for the RHOA negative cases, suggesting that other genetic mutations in $R H O A$ mutated cases may be more damaging. This could indicate that $R H O A$ 
mutations may be more oncogenic and result in increased mutations due to increased DNA damage. Alternatively, it is possible that cases with $R H O A$ mutations require more damaging co-mutations in order for oncogenesis to take form. Further in vitro and in vivo investigation is therefore required to assess these possibilities.

The present study also assessed samples for COSMIC signatures seen in B-cell lymphomas and solid tumors (Fig. S4). Some broad COSMIC signatures that are seen in most tumors were also present in our samples, and no COSMIC signature was unique to RHOA mutational status. In addition, a COSMIC T-cell lymphoma tumor signature was not present in the COSMIC database. Generating such a signature by using multiple T-cell lymphoma subtypes may be required.

Although the mutational burden was higher in the RHOA positive cases (8.2) compared with the RHOA negative cases (2.4), which was consistent with a previous study by Sakata-Yanagimoto et al (7), the present study reported a higher mutational burden in promoter regions and CTCF binding sites for the $R H O A$ negative group compared with the RHOA positive group. Promoter regions and CTCF binding sites serve crucial roles in the regulation of gene transcription and expression $(45,46)$. In the present study, RHOA negative cases seemed to present with a more heterogenous mutational landscape, which makes them difficult to describe and classify. These mutations may affect common pathways that ultimately lead to an AITL phenotype, even in the absence of a RHOA mutation. This dysregulation of yet unidentified pathways may also happen in other non-coding areas. In order to identify the promoter regions or CTCF binding sites regulating the oncogenesis in the RHOA negative or positive cases, CHIP-Seq or functional studies would be required in the future.

AITL is often associated with EBV infected cells and EBV is identified primarily in non-T-cells (the B-cells) (47); however, only a few studies evaluated the role of EBV in AITL pathology. In the present study, six out of 10 patients had a positive EBV infection status following NGS and ISH analysis. When looking at the gene expression of our cases with regards to EBV infected cells, there was no difference in the gene expression profile between positive and negative cases. After comparison of the mutational profiles of our cases, a higher mutational burden was observed in the EBV negative cases. However, the average CADD score was higher in the EBV positive cases, suggesting that an EBV infection may contribute to a more deleterious mutational scenery. Previous studies suggested that a contribution to T-cell lymphomagenesis is excluded as there was no virus found in the neoplastic T-cells $(48,49)$; however, it was reported that an EBV infection in AITL cases could lead to histologic progression of these cases (49). A previous study investigating 270 cases of AITL reported that in young patients with AITL, an EBV infection was associated with a significantly improved prognosis (50). Kaplan-Meier Survival analysis was performed with regards to EBV infection, and the results demonstrated no improved overall survival for EBV positive cases. Our results do not confirm these previous findings of Eladl et al (50) but our sample size is small. Another study by Hoffman et al (51) assessed the association between EBV infection and diffuse large B-cell lymphoma (DLBCL) occurring in PTCL, and reported that patients with PTCL and DLBCL frequently have
EBV infected B-cells, suggesting an important role of EBV in B-cell transformation.

The immunologic environment of AITL is characterized by a massive infiltration of inflammatory cells (52). An analysis of immune cell infiltration within our AITL cases based on gene expression profiling was performed. The results demonstrated that the greatest contributors to the gene expression profiles were naïve B-cells, resting memory CD4 T-cells and T-follicular helper cells. Amongst the RHOA positive cases, the portion of naïve B-cells and T-follicular helper cells seemed to be elevated compared with other cases. Previous studies investigating the gene expression profiles of AITL reported an overexpression of T-follicular helper cells in AITL, since these are the neoplastic infiltrates, and of vascular endothelial growth factor $(53,54)$. Nguyen et al $(55)$ identified mutations specific to both T-cell and B-cells within nodal T-cell lymphomas.

Although the present study only included 10 patients with AITL, we were interested in assessing statistics associated with the prognosis of patients with AITL. The relapse-free survival and overall survival were evaluated and compared by RHOA mutational status. The results demonstrated that $R H O A$ positive cases had a better overall survival and relapse-free survival $(\mathrm{P}$-value $=0.048)$, although these cases tend to have an increased mutational burden. However, improved outcomes may be due to improved immune system surveillance in the setting of more tumor antigenic stimulation. Further studies are needed to assess this possibility.

Single cell imaging analysis demonstrated that $R H O A$ mutation positive cells may be more circular than $R H O A$ mutation negative cells, which, based on the known function of RHOA, could be a consequence of altered actin dynamics. This difference was not significant, but an increased variation between these subtypes may be observed by increasing the total sample size, and a greater variance may also be detected with respect to circularity $(36,37)$. However, it is important to acknowledge that the mutational status of RHOA in the individual cells was unclear, since they were selected randomly. A single cell NGS study is therefore required to confirm the absolute mutational status in these cells.

In conclusion, the results from the present study demonstrate the differences and similarities of RHOA mutated and non-mutated AITL, identified both increased burden and deleterious mutations in coding and non-coding (promoter and transcription binding site) regions of RHOA mutated AITLs, highlighted differences in the immune system infiltrate, as well as a specific single cell morphologic manifestation in cases of AITL (increased circularity). Further studies are needed to investigate the immunologic environment in patients with AITL in the context of understanding RHOA mutations and their cell biologic responses that will be important future avenues of study.

\section{Acknowledgements}

Not applicable.

\section{Funding}

This study was supported through grant funding (grant no. 1187454) from Agilent Technologies. 


\section{Availability of data and materials}

The datasets used and/or analyzed during the current study are available from the corresponding author on reasonable request.

\section{Authors' contributions}

$\mathrm{RO}, \mathrm{AB}, \mathrm{MKS}, \mathrm{RW}, \mathrm{BP}$ and ER designed the research. RSO and $\mathrm{AB}$ wrote the paper. DJ and AB performed analyses. RSO, $\mathrm{AB}, \mathrm{DJ}, \mathrm{KS}$ and JK analyzed data and edited the paper. NS and AB performed research. ER provided expertise. All authors read and approved the final manuscript.

\section{Ethics approval and consent to participate}

This study was approved by the Ethics approval from Stanford University's Institutional Review Board (approval no. IRB-22359).

\section{Patient consent for publication}

Not applicable.

\section{Competing interests}

This work was supported by funding from Agilent Technologies to RO, and ER is an employee of Agilent Technologies. Other authors declare that they have no competing interest.

\section{References}

1. Attygalle AD, Kyriakou C, Dupuis J, Grogg KL, Diss TC, Wotherspoon AC, Chuang SS, Cabeçadas J, Isaacson PG Du MQ, et al: Histologic evolution of angioimmunoblastic T-cell lymphoma in consecutive biopsies: Clinical correlation. Am J Surg Pathol 31: 1077-1088, 2007.

2. Dogan A, Atygalle AD and Kyriakou C: Angioimmunoblastic T-cell lymphoma. Br J Haematol 121: 681-691, 2003.

3. Attygalle A, Al-Jehani R, Diss TC, Munson P, Liu H, Du MQ, Isaacson PG and Dogan A: Neoplastic T cells in angioimmunoblastic T-cell lymphoma express CD10. Blood 99: 627-633, 2002

4. Manso R, Sánchez-Beato M, Monsalvo S, Gómez S, Cereceda L, Llamas P, Rojo F, Mollejo M, Menárguez J, Alves J, et al: The RHOA G17V gene mutation occurs frequently in peripheral T-cell lymphoma and is associated with a characteristic molecular signature. Blood 123: 2893-2894, 2014.

5. Cairns RA, Iqbal J, Lemonnier F, Kucuk C, de Leval L, Jais JP, Parrens M, Martin A, Xerri L, Brousset P, et al: IDH2 mutations are frequent in angioimmunoblastic T-cell lymphoma. Blood 119: 1901-1903, 2012.

6. Enami T, Yoshida K, Shiraishi Y, Ishii R, Miyake Y, Muto H, Tsuyama N, Okuno Y, Sakata S, Kamada Y, et al: Somatic G17V Rhoa mutation specifies angioimmunoblastic T-Cell lymphoma. Blood 122: 815, 2013.

7. Sakata-Yanagimoto M, Enami T, Yoshida K, Shiraishi Y, Ishii R, Miyake Y, Muto H, Tsuyama N, Sato-Otsubo A, Okuno Y, et al Somatic RHOA mutation in angioimmunoblastic $\mathrm{T}$ cell lymphoma. Nat Genet 46: 171-178, 2014.

8. Fujisawa M, Sakata-Yanagimoto M, Nishizawa S, Komori D, Gershon P, Kiryu M, Tanzima S, Fukumoto K, Enami T, Muratani M, et al: Activation of RHOA-VAV1 signaling in angioimmunoblastic T-cell lymphoma. Leukemia 32: 694-702, 2018.

9. Cortes JR, Ambesi-Impiombato A, Couronné L, Quinn SA, Kim CS, da Silva Almeida AC, West Z, Belver L, Martin MS, Scourzic L, et al: RHOA G17V induces T follicular helper cell specification and promotes lymphomagenesis. Cancer Cell 33: 259-273.e7, 2018.
10. Ondrejka SL, Grzywacz B, Bodo J, Makishima H, Polprasert C, Said JW, Przychodzen B, Maciejewski JP and His ED: Angioimmunoblastic T-cell lymphomas with the RHOA p. Gly17Val mutation have classic clinical and pathologic features. Am J Surg Pathol 40: 335-341, 2016.

11. Swerdlow SH, Campo E, Pileri SA, Harris NL, Stein H, Siebert R, Advani R, Ghielmini M, Salles GA, Zelenetz AD and Jaffe ES: The 2016 revision of the World Health Organization classification of lymphoid neoplasms. Blood 127: 2375-2391, 2016.

12. Swerdlow SH, Campo E, Harris NL, Jaffe ES, Pileri SA, Stein $\mathrm{H}$ and Thiele $\mathrm{J}$ (eds): WHO Classification of Tumours of Haematopoietic and Lymphoid Tissues. Revised 4th edition. International Agency for Research in Cancer (IARC), Lyon, 2017.

13. Kumar J, Butzmann A, Wu S, Easly S, Zehnder JL, Warnke RA, Bangs CD, Jangam D, Cherry A, Lau J, et al: Indolent in situ B-cell neoplasms with MYC rearrangements show somatic mutations in MYC and TNFRSF14 by next-generation sequencing. Am J Surg Pathol 43: 1720-1725, 2019.

14. McKenna A, Hanna M, Banks E, Sivachenko A, Cibulskis K, Kernytsky A, Garimella K, Altshuler D, Gabriel S, Daly M and DePristo MA: The Genome Analysis Toolkit: A MapReduceframework for analyzing next-generation DNA sequencing data. Genome Res 20: 1297-1303, 2010.

15. Koboldt DC, Zhang Q, Larson DE, Shen D, McLellan MD, Lin L, Miller CA, Mardis ER, Ding L and Wilson RK: VarScan2: Somatic mutation and copy number alteration discovery in cancerby exome sequencing. Genome Res 22: 568-576, 2012.

16. Karczewski KJ, Francioli LC, Tiao G, Cummings BB, Alföldi J, Wang Q, Collins RL,Laricchia KM, Ganna A, Birnbaum DP, et al: The mutational constraint spectrum quantified from variation in 141,456 humans. Nature 581: 434-443, 2020.

17. Rehm HL, Berg JS, Brooks LD, Bustamante CD, Evans JP, Landrum MJ, Ledbetter DH, Maglott DR, Martin CL, Nussbaum RL, et al: ClinGen-the clinical genome resource. N Engl J Med 372: 2235-2242, 2015.

18. 1000 Genomes Project Consortium; Auton A, Brooks LD, Durbin RM, Garrison EP, Kang HM, Korbel JO, Marchini JL, McCarthy S, McVean GA and Abecasis GR: A global reference for human genetic variation. Nature 526: 68-74, 2015.

19. Exome Variant Server, NHLBI GO Exome Sequencing Project (ESP), Seattle, WA. http://evs.gs.washington.edu/EVS/. Accessed February 16, 2020

20. Kopanos C, Tsiolkas V, Kouris A Chapple CE, Albarca Aguilera M, Meyer R and Massouras A: VarSome: The human genomic variant search engine. Bioinformatics 35: 1978-1980, 2019.

21. Blokzijl F, Janssen R, Van Boxtel R and Cuppen E: MutationalPatterns: Comprehensive genome-wide analysis of mutational processes. Genome Med 10: 33, 2018.

22. Chen EY, Tan CM, Kou Y, Duan Q, Wang Z, Meirelles GV, Clark NR and Ma'ayan A: Enrichr: Interactive and collaborative HTML5 gene list enrichment analysis tool. BMC Bioinformatics 14: 128, 2013.

23. Subramanian A, Tamayo P, Mootha VK, Mukherjee S, Ebert BL, Gillette MA, Paulovich A, Pomeroy SL, Golub TR, Lander ES and Mesirov JP: Gene set enrichment analysis: A knowledge-based approach for interpreting genome-wide expression profiles. Proc Natl Acad Sci USA 102: 15545-15550, 2005.

24. Mootha VK, Lindgren CM, Eriksson KF, Subramanian A, Sihag S, Lehar J, Puigserver P, Carlsson E, Ridderstråle M, Laurila E, et al: PGC-1alpha-responsive genes involved in oxidative phosphorylation are coordinately downregulated in human diabetes. Nat Genet 34: 267-273, 2003.

25. Kamburov A, Stelzl U, Lehrach $\mathrm{H}$ and Herwig R: The ConsensusPathDB interaction database: 2013 update. Nucleic Acids Res 41 (Database Issue): D793-D800, 2013.

26. Gyarmati P, Kjellander C, Aust C, Song Y, Öhrmalm L and Giske CG: Metagenomic analysis of bloodstream infections in patients with acute leukemia and therapy-induced neutropenia. Sci Rep 21: 23532, 2016.

27. Baheti S, Tang X, O'Brien DR, Chia N, Roberts LR, Nelson H, Boughey JC, Wang L, Goetz MP, Kocher JA and Kalari KR: HGT-ID: An efficient and sensitive workflow to detect human-viral insertion sites using next-generation sequencing data. BMC Bioinformatics 19: 271, 2018.

28. Kim D, Paggi JM, Park C, Bennett C and Salzberg SL: Graph-based genome alignment and genotyping with HISAT2 and HISAT-genotype. Nat Biotechnol 37: 907-915, 2019.

29. Anders S, Pyl PT and Huber W: HTSeq-a Python framework to work with high-throughoutput sequencing data. Bioinformatics 31: 166-169, 2015. 
30. R Studio Team. R Studio: Integrated Development for R. Version 1.2.5001. Mode: desktop. RStudio, PBC, Boston, MA, 2020. http://www.rstudio.com/. Accessed February 16, 2020.

31. Metsalu T and Vilo J: ClustVis: A web tool visualizing clustering of multivariate data using Prinicpal Component Analysis and heatmap. Nucleic Acids Res 43 (W1): W566-W570, 2015.

32. Newman AM, Liu CL, Green MR, Gentles AJ, Feng W, Xu Y, Hoang CD, Diehn $M$ and Alizadeh AA: Robust enumeration of cell subsets from tissue expression profiles. Nat Methods 12: 453-457, 2015.

33. Jangam D, Sridhar K, Butzmann A, Sambhagati P, Plowey ED and Ohgami RS: TBL1XR1 Mutations in primary marginal zone lymphomas of occular adnexa are associated with unique morphometric phenotypes. Curr Eye Res: Jun 19, 2020 (Epub ahead of print)

34. Wong MA and Hartigan JA: Algorithm AS 136: A K-means clustering algorithm. J R Stat Soc Ser C 28: 100-108, 1979.

35. Fouad S, Randell D, Galton A, Mehanna H and Landini G: Unsupervised morphological segmentation of tissue compartments in histopathological images. PLoS One 12: e0188717, 2017.

36. Hart M, Lauer J, Selig M, Hanak M, Walters B and Rolauffs B: Shaping the cell and the future: Recent advancements in biophysical aspects relevant to regenerative medicine. J Funct Morphol Kinesiol 3: 2, 2017.

37. Lyons SM, Alizadeh E, Mannheimer J, Schuamberg K, Castle J, Schroder B, Turk P, Thamm D and Prasad A: Changes in cell shape are correlated with metastatic potential in murine and human osteosarcomas. Biol Open 5: 289-299, 2016.

38. Chiba S, Enami T, Ogawa S and Sakata-Yanagimoto M: G17V RHOA: Genetic evidence of GTP-unbound RHOA playing a role in tumorigenesis in T cells. Small GTPases 6: 100-103, 2015.

39. Nagao R, Kikuti YY, Carreras J, Kikuchi T, Miyaoka M, Matsushita H, Kojima M, Ando K, Sakata-Yanagimoto M, Chiba S and Nakamura N: Clinicopathologic analysis of angioimmunoblastic T-cell lymphoma with or without RHOA G17V mutation using formalin-fixed paraffin-embedded sections. Am J Surg Pathol 40: 1041-1050, 2016.

40. Willemsen M, Hamid MA, Winkens B and Zur Hausen A: Mutational heterogeneity of angioimmunoblastic T-cell lymphoma indicates distinct lymphomagenic pathways. Blood Cancer J 8: 6, 2018.

41. Yoo HY, Sung MK, Lee SH, Kim S, Lee H, Park S, Kim SC, Lee B, Rho K, Lee JE, et al: A recurrent inactivating mutation in RHOA GTPase in angioimmunoblastic T cell lymphoma. Nat Genet 46: 371-375, 2014.

42. Wang M, Zhang S, Chuang SS, Ashton-Key M, Ochoa E, Bolli N, Vassiliou G, Gao Z and Du MQ: Angioimmunoblastic T cell lymphoma: Novel molecular insights by mutation profiling. Oncotarget 8: 17763-17770, 2017.

43. Ng SY, Brown L, Stevenson K, deSouza T, Aster JC, Louissaint A Jr and Weinstock DM: RhoA G17V is sufficient to induce autoimmunity and promotes T-cell lymphomagenesis in mice. Blood 132: 935-947, 2018.

44. Abate F, Da Silva-Almeida AC, Zairis S, Zairis S, Robles-Valero J, Couronne L, Khiabanian H, Quinn SA, Kim MY, Laginestra MA, et al: Activating mutations and translocations in the guanine exchange factor VAV1 in peripheral T-cell lymphomas. Proc Natl Acad Sci USA 114: 764-769, 2017.
45. Cornish AJ, Hoang PH, Dobbins SE, Law PJ, Chubb D, Orlando G and Houlston RS: Identification of recurrent non-coding mutations in B-cell lymphoma using Hi-C. Blood Adv 3: 21-32, 2019.

46. Zhang X, Zhang Y, Ba Z, Kyritsis N, Casellas R and Alt FW: Fundamental roles of chromatin loop extrusion in antibody class switching. Nature 575: 385-389, 2019.

47. Weiss LM, Jaffe ES, Liu XF, Chen YY, Shibata D and Medeiros LJ: Detection and localization of Epstein-Barr viral genomes in angioimmunoblastic lymphadenopathy and angioimmunoblastic lymphadenopathy-like lymphoma. Blood 79: 1789-1795, 1992.

48. Khan G, Norton AJ and Slavin G: Epstein-Barr virus in angioimmunoblastic T-cell lymphomas. Histopathology 22: 145-149, 1993.

49. Zhou Y, Attygalle AD, Chuang SS, Diss T, Ye H, Liu H, Hamoudi RA, Munson P, Bacon CM, Dogan A and Du MQ: Angioimmunoblastic T-cell lymphoma: Histological progression associates with EBV and HHV6B viral load. Br J Haematol 138: 44-53, 2007.

50. Eladl AE, Shimada K, Suzuki Y, Takahara T, Kato S, Kohno K, Elsayed AA, Wu CC, Tokunaga T, Kinoshita T, et al: EBV status has prognostic implication among young patients with angioimmunoblastic T-cell lymphoma. Cancer Med 9: 678-688, 2020.

51. Hoffmann JC, Chisholm KM, Cherry A, Chen J, Arber DA, Natkunam Y, Warnke RA and Ohgami RS: An analysis of MYC and EBV in diffuse large B-cell lymphomas associated with angioimmunoblastic T-cell lymphoma and peripheral T-cell lymphoma not otherwise specified. Hum Pathol 48: 9-17, 2016

52. Bal M, Gujral S, Gandhi J, Shet T, Epari S and Subramanian PG: Angioimmunoblastic T-cell lymphoma: A critical analysis of clinical, morphologic and immunophenotypic features. Indian J Pathol Microbiol 53: 640-645, 2010.

53. De Leval L, Rickman DS, Thielen C, Reynies Ad, Huang YL, Delsol G, Lamant L, Leroy K, Brière J, Molina T, et al: The gene expression profile of nodal peripheral T-cell lymphoma demonstrates a molecular link between angioimmunoblastic T-cell lymphoma (AITL) and follicular helper T (TFH) cells. Blood 109: 4952-4963, 2007.

54. Piccaluga PP, Agostinelli C, Califano A, Carbone A, Fantoni L, Ferrari S, Gazzola A, Gloghini A, Righi S, Rossi M, et al: Gene expression analysis of angioimmunoblastic lymphoma indicates derivation from $\mathrm{T}$ follicular helper cells and vascular endothelial growth factor deregulation. Cancer Res 67: 10703-10713, 2007.

55. Nguyen TB, Sakata-Yanagimoto M, Asabe Y, Matsubara D, Kano J, Yoshida K, Shiraishi Y, Chiba K, Tanaka H, Miyano S, et al: Identification of cell-type-specific mutations in nodal T-cell lymphomas. Blood Cancer J 7: e516, 2017.

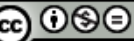

This work is licensed under a Creative Commons Attribution-NonCommercial-NoDerivatives 4.0 International (CC BY-NC-ND 4.0) License. 